\title{
Neurosurgical planning in a low-resource setting using free open-source three-dimensional volume-rendering software
}

\author{
Oliver Ryan M. Malilay, MD, Kevin Paul Ferraris, MD, MBA, and Joseph Erroll V. Navarro, MD \\ Department of Surgery, Jose R. Reyes Memorial Medical Center, Manila, Philippines
}

$\mathrm{P}$ LANNING how to conduct neurosurgery is a daunting task, especially for trainees and young neurosurgeons. Inexperience can result in inappropriate incisions, inadequate craniotomies, and difficult surgeries. As early as 1998, neurosurgeons developed the means of creating three-dimensional (3D) reconstructions based on imaging technologies to address this problem. ${ }^{1}$ Neuronavigational systems, such as Stealth (Medtronic) and Curve (Brainlab), are useful adjuncts that enable accurate (mean error $<1.5 \mathrm{~mm}$ ) surgical planning as well as real-time intraoperative orientation. ${ }^{2}$ Unfortunately, these systems are often expensive and not readily available to all institutions, especially those in low- to middle-income countries.

Other navigational workstations devoted solely to surgical planning may be effective, but they are bulky and require dedicated areas in the hospital where they can be used. $^{3-7}$ Commercial software, such as OsiriX (Pixmeo) and radiANT (Medixant), are available and have been shown to be useful in 3D reconstruction of cortical topography, ${ }^{8}$ volume rendering, ${ }^{9}$ and preoperative planning of neurosurgical cases..$^{10}$ Unfortunately, institutions without adequate resources cannot afford licenses for their usethus the need for low-cost, readily available software. In this editorial, we describe how we were able to use Horos (Horos Project), a free, open-source, readily downloadable program for planning cranial neurosurgery.

\section{Program Description and Interface}

Imaging manipulation was performed using Horos, which can be downloaded from https://www.horosproject. org/download at no cost to the user. The program automatically loads digital imaging in communications in medicine (DICOM) files when a CD/DVD is inserted into the computer, or DICOM files can be imported manually from flash drives. The default $2 \mathrm{D}$ viewer allows basic tasks such as viewing, magnifying, and manipulating the window level of the images, and can also be used for more advanced functions such as performing exact measurements (even those involving curved surfaces) and marking regions of interest (ROIs), which are used for computing volumes and creating tumor models. On the other hand, the $3 \mathrm{D}$ viewer can be used to create 3D models of patients and perform virtual craniotomies that are suitable for preoperative planning.

\section{Preoperative Planning}

A major advantage of Horos software lies in the ability to rotate the generated 3D models to simulate patients' positions during surgery, after which a virtual craniotomy can be made according to the shape and size desired by the surgeon. With this capability, various operative trajectories can be evaluated until an appropriate approach is chosen. For cerebrovascular cases, this has enabled us to study the aneurysm's relationship to surrounding bone, anticipate major vessels and their orientations as we encounter them, visualize aneurysm morphology, approximate the postdissection microscopic perspective, and predict which clips would most likely be used. Similarly, use of the program for planning tumor surgery allows accurate measurements of tumor size and volume as well as the creation of an in situ 3D model of the tumor in its correct spatial position, which then allows for the plotting of suitable incisions and craniotomies.

\section{Clinical Utility}

Measurements made using software for the 3D reconstruction of images from DICOM files have been shown to be accurate to $0.1 \mathrm{~mm} .{ }^{11}$ Imaging manipulation software programs have been shown to have $98 \%$ sensitivity and $100 \%$ specificity for detecting ruptured intracranial aneurysms..$^{12}$ In addition to these advantages, Horos can also be used to help predict appropriate approaches to aneurysms, such as whether an anterior clinoidectomy might be required for a low-lying posterior communicating artery 
aneurysm or if the projection of an anterior communicating artery aneurysm is favorable for a lateral supraorbital approach. For tumor cases, combining ROI formation and cortical topography recreation can assist in planning resection trajectories by establishing tumor boundaries or identifying sulci for corticectomy. ${ }^{13}$

An additional advantage conferred by the use of Horos is that it allows senior consultants to facilitate training in a remote manner such as through "virtual planning sessions," during which they can discuss the conduct of the surgery through videoconferencing. The use of Horos can also mitigate the decrease in opportunities to perform or assist in surgery, and hasten the acquisition of skills when learning how to perform new operations. This is particularly important for institutions in low- and middle-income countries that may not have access to other options for operative planning. Finally, an additional benefit of using this application is seen in the informed consent process: by showing patients the 3D models generated by Horos, patients are better able to understand where their lesion lies, what size it is, and how their operation will proceed. This increased comprehension on the part of the patient has the potential to foster trust in the surgeon and increase patient satisfaction.

\section{Conclusions}

Neurosurgical planning using free open-source software allows surgeons to preoperatively reconstruct patients' anatomies, highlight their pathological conditions, and create a safe and appropriate surgical plan without costly equipment. This can be used by both residents and young neurosurgeons at the start of their careers or as a teaching tool by experienced practitioners with complicated cases.

https://thejns.org/doi/abs/10.3171/2020.10.FOCUS20772

\section{References}

1. Chabrerie A, Ozlen F, Nakajima S, et al. Three-dimensional image reconstruction for low-grade glioma surgery. Neurosurg Focus. 1998;4(4):E7.

2. Paraskevopoulos D, Unterberg A, Metzner R, et al. Comparative study of application accuracy of two frameless neuronavigation systems: experimental error assessment quantifying registration methods and clinically influencing factors. Neurosurg Rev. 2010;34(2):217-228.

3. de Notaris M, Palma K, Serra L, et al. A three-dimensional computer-based perspective of the skull base. World Neurosurgery. 2014;82(6 Suppl):S41-S48.
4. Gong XY, Higano S, Mugikura S, et al. Virtually peeling off the skull and scalp: a simple way of mapping the superficial cerebral veins on the brain surface. Stereotact Funct Neurosurg. 2008;86(6):345-350.

5. Oishi M, Fukuda M, Ishida G, et al. Presurgical simulation with advanced 3 -dimensional multifusion volumetric imaging in patients with skull base tumors. Neurosurgery. 2011; 68(1 Suppl Operative):ons188-ons199.

6. Beyer J, Hadwiger M, Wolfsberger S, Buhler K. High-quality multimodal volume rendering for preoperative planning of neurosurgical interventions. IEEE Trans Visual Comput Graphics. 2007;13(6):1696-1703.

7. Aydin K, Cokluk C, Kuruoglu E, et al. Using the magnetic resonance three-dimensional volume rendering for tissues technique in the planning of craniotomy flaps with linear scalp incision. Minim Invasive Neurosurg. 2006;49(4): 189-193.

8. Harput MV, Gonzalez-Lopez P, Türe U. Three-dimensional reconstruction of the topographical cerebral surface anatomy for presurgical planning with free OsiriX software. Neurosurgery. 2014;10(Suppl 3):426-435.

9. Jha DK, Khera P, Bhaskar S, Garg M. Three-dimensional volume rendering: an underutilized tool in neurosurgery. World Neurosurgery. 2019;130:485-492.

10. Spiriev T, Nakov V, Laleva L, Tzekov C. OsiriX software as a preoperative planning tool in cranial neurosurgery: a stepby-step guide for neurosurgical residents. Surg Neurol Int. 2017;8(1):241.

11. Kim G, Jung H-J, Lee H-J, et al. Accuracy and reliability of length measurements on three-dimensional computed tomography using open-source OsiriX software. J Digit Imaging. 2012;25(4):486-491.

12. Wang Y-C, Liu Y-C, Hsieh T-C, et al. Aneurysmal subarachnoid hemorrhage diagnosis with computed tomographic angiography and OsiriX. Acta Neurochir. 2010;152(2): 263-269.

13. Lovato R, Araujo JV, Paiva AC, et al. The use of OsiriX for surgical planning using cranial measures and region of interest tools: technical note. Asian J Neurosurg. 2019;14(3): $762-766$.

\section{Disclosures}

The authors report no conflict of interest.

\section{Correspondence}

Oliver Ryan M. Malilay: olivermalilay@gmail.com.

\section{INCLUDE WHEN CITING}

DOI: $10.3171 / 2020.10$. FOCUS20772. 